\title{
Antonio Porras-Nadales. La acción de gobierno. Gobernabilidad, gobernanza, gobermedia. Madrid, Editorial Trotta, 2014 (148 páginas).
}

La acción de gobierno permite conocer la posición de las instituciones gubernamentales respecto de los problemas de la sociedad. Esto es particularmente complejo dentro del contexto que marcan la globalización, las crisis y las incertidumbres que plantean. Constituye un reto trazar modelos que plasmen la evolución del Estado intervencionista. Para ello, se debe afrontar la tarea de determinar la posición de los gobiernos según los enfoques, estrategias y herramientas que configuran su acción.

El autor comienza dibujando un contexto histórico en el que manifiesta las diferencias históricas de la acción de gobierno del Estado liberal abstencionista y social intervencionista. El gobierno del Estado liberal se presenta como un fin y no como un medio para ejercer la acción de gobierno, mero ejecutor de las leyes. El periodo de transición del s. XIX al s. XX estuvo marcado por la lucha de clases. En esta etapa los gobiernos empiezan a jugar cada vez más un papel político.

Aparece el Estado social, con un gobierno autónomo que interviene para asegurar un proceso social pacífico y garantizar los derechos y obligaciones constitucionales. Se pone en marcha una auténtica acción de gobierno. Este modelo introduce los derechos sociales, que incrementan las demandas de la ciudadanía.

El Estado social entra en crisis por la sobrecarga de la agenda del Gobierno, debida al exceso de demanda social y déficit de oferta pública, traducido en un exceso de gasto público y un déficit de ingresos. La acción de gobierno se ve condicionada por partidas presupuestarias más limitadas y la creciente intervención empieza a encontrar dificultades para legitimarse.

Posteriormente surge la democracia avanzada, en la que el Poder Ejecutivo necesita consensuar sus decisiones con el resto de actores. Las demandas sociales se hacen más complejas y son, a menudo, contradictorias, por lo que la acción de gobierno no puede ser ya puramente racional. El color del gobierno es secundario, así como las finalidades de su acción de gobierno, marcadas por los valores constitucionales. Es en el diseño y orientación de la acción del gobierno donde radica la importancia.

La agenda del gobierno permite distribuir en el tiempo las acciones (y las no-acciones). La no-acción puede tener un sentido propio, aparte de la no disponibilidad de los recursos para llevar a cabo la acción. La agenda permite la adecuación del programa de gobierno a las demandas coyunturales. Las "capacidades" del gobierno definen los tipos de problemas a los que éste debe hacer frente. La agenda gubernamental está limitada por la "sobrecarga" del Estado social y por los cortos periodos de las legislaturas (mandato).

El autor muestra los enfoques del análisis que pretende realizar: estructural-instrumental (relacionado con la gobernabilidad), funcional-estratégico (gobernanza) y simbólico-mediática (gobermedia).

El segundo capítulo trata sobre la gobernabilidad. El autor destaca, en primer lugar, la importancia de los gobiernos estables en la consolidación de las democracias modernas y, eventualmente, en el desarrollo de la acción del gobierno. Como factores para la estabilidad se señalan el sistema de partidos, por un lado, y la plasmación 
constitucional de las distintas formas de gobierno, por otro. En relación al primero de los factores, el autor refleja la evolución de los sistemas de partidos durante el siglo XX: de los sistemas atomizados a sistemas más estables como el bipartidista, gracias a la aparición de los partidos catch-all. Resultaría interesante comprobar cómo, en la actualidad, la crisis de los sistemas "estables" puede afectar a la acción del gobierno. En cuanto al segundo factor, sobresalen los mecanismos parlamentarios, que garantizan una mayor estabilidad gubernamental, por ejemplo como puede ser la moción de censura constructiva.

Garantizado un gobierno estable, uno de los retos que se le planteó al Estado intervencionista fue el de implementar políticas que desarrollasen los nuevos valores sociales, que consagraban las constituciones de la época. El Estado comienza a intervenir en la economía mediante la nacionalización de sectores estratégicos, lo que hizo tambalear el principio de libertad de empresa. Se apoyaba para ello en la idea de planificación y en una pujante tecnocracia, que propició el desarrollismo económico. Pero, con la crisis petrolífera, las disfuncionalidades económicas y cierto auge del liberalismo, este modelo daría paso a otro caracterizado por el impulso o indirizzo político, como remarca Porras-Nadales. Los gobiernos ya no se limitan a la mera administración, sino que el impulso político les permite dejar su impronta en la acción de gobierno que llevan a cabo. Esta acción de gobierno estará marcada, a partir de este momento, por los programas de gobierno, que establecerán las prioridades del partido gobernante. Todo esto entraría en conflicto con la tradicional burocracia.

El modelo administrativo resultante, a la manera de Easton, se nutría de unos inputs para desarrollar unos outputs encaminados a satisfacer las necesidades públicas, conformando así la acción de gobierno. Los valores sociales de las constituciones tendrían su respuesta en la creación del concepto de servicio público. El autor se plantea el funcionamiento del circuito de inputs y outputs, en relación a la teoría del impulso político. La entrada de demandas sociales está coartada por la impermeabilidad de los partidos y éstos suelen configurar las demandas según les convenga en términos de marketing electoral. En cuanto a las salidas, se comprueba que el modelo de impulso político no se adapta adecuadamente al marco establecido por el Estado del Bienestar, ya que se deben tener en cuenta múltiples factores en las tomas de decisiones para los que el modelo del indirizzo no resulta el más apropiado.

En el tercer capítulo, Porras-Nadales presenta la gobernanza como nuevo paradigma intervencionista en sustitución de la gobernabilidad. El anterior paradigma, que estaba marcado por la consolidación de la democracia, da paso a la democracia avanzada, que exige una mayor calidad y eficiencia de la acción del gobierno. La intervención comienza a enfocarse desde la perspectiva de las políticas públicas, que incorpora técnicas de gestión que hasta el momento eran más propias de la esfera privada. Los gestores públicos incluyen en los procesos de diseño e implementación de las políticas públicas a los actores afectados y a técnicos especializados en la materia a tratar. Se pasa así de un procedimiento esencialmente vertical, propio del impulso político, a otro más vertical. Este mayor protagonismo de las redes de la sociedad provoca cierto alejamiento del debate de las políticas públicas de sus tradicionales escenarios, como el parlamentario.

Con el modelo de la gobernanza, el programa del partido gobernante pierde importancia en favor de las demandas sociales a las que se trata de dar respuesta. El proceso comenzaría desde la periferia, donde existe mayor permeabilidad para las demandas, hasta la final fijación de éstas en las agendas. En este contexto de auge de los actores sociales y los expertos, el consenso adquiere gran relevancia 
y los gobiernos de distintos niveles territoriales pierden autonomía para definir sus agendas, ya que deben contar con los anteriores actores mediante la cooperación y coordinación con ellos. Los gobiernos pasan a tener una función meramente orientadora o implementadora de las políticas públicas, lo que provoca que sus funciones de dirección y programación disminuyan.

Porras-Nadales también señala el papel subsidiario que los gobiernos centrales tienen en el modelos de la gobernanza, ya que los procesos de interacción se darían en gobiernos de menor nivel territorial, al estar más próximos a las demandas sociales, adquiriendo los primeros un rol de integrador de todos esos procesos ("gobernanza sin gobierno"). El modelo de la gobernanza no está exento de complicaciones. Por un lado, el control del cumplimiento objetivos concretos se lleva a cabo mediante la evaluación, pero mucho más difíciles de evaluar son las "metafunciones orientadoras del gobierno", como apunta el autor. Por otro lado, existe un riesgo lógico de "captura" de los procesos participativos, cuando intereses estructurados desplazan del debate a otros grupos. Además, la gobernanza multinivel requiere una organización institucional particularmente compleja, debido a las necesidades de cooperación y coordinación de los distintos niveles institucionales entre sí y de éstos con las distintas redes sociales. Si la gobernanza multinivel implica el desplazamiento de las decisiones hacia múltiples instituciones gubernamentales, la responsabilidad política se dispersa, haciéndola difícilmente identificable. El autor concluye que la persistencia de cierta cultura de la gobernabilidad, unida al lento y asimétrico desarrollo del modelo de la gobernanza, ha impedido su desarrollo efectivo.

En el cuarto capítulo, el politólogo explica que el vacío en la esfera representativa provocado por la gobernanza, vuelve a ser ocupado por la política competitiva. Se busca volver a controlar la centralidad mediante la competición en la arena política desde la cual ahora se generan los impulsos. En este momento adquiere gran importancia quién gobierna y la disputa por ocupar la centralidad se escenifica enormemente en los medios de comunicación. El autor acuña el término de "gobermedia" para este nuevo modelo de la acción del gobierno, caracterizada por dirigirse principalmente a su difusión en los medios, lo que suscita respuestas inmediatas que pueden condicionar la agenda. La imagen y el liderazgo, que ya con la gobernanza adquirió relevancia por su utilidad en los procesos negociadores, son de suma importancia por la proyección mediática. Se adquiere, con los nuevos medios de comunicación, una percepción instantánea de la acción del gobierno. La visualización del gobernante se transforma en un elemento legitimador a ojos de la opinión pública. Pero ésta es un arma de doble filo, ya que en el caso de políticas deficientes podría tener el efecto contrario.

La gobermedia también facilita el pluralismo, pues acoge a gran número de alternativas, permitiendo la difusión de todo tipo de críticas a la gestión de los gobiernos. La competencia entre partidos se desenvuelve en este modelo en el campo de la retórica. Esto produce el florecimiento de los "partidos cártel", aquéllos que disponen de gran apoyo mediático. El autor se plantea dónde se ubica la gobermedia, formulando dos hipótesis: si se ha integrado con la gobernanza, complementándola y agilizando el procedimiento intervencionista; o, por el contrario, es autónoma y ha perdido sus vínculos con el procedimiento intervencionista.

Con la gobermedia, el diseño de la acción de gobierno corre el riesgo de perder la racionalidad científica al poner el foco en unos problemas y no en otros, normalmente atendiendo a criterios electoralistas. Las comparecencias se convierten en el método por el que los gobernantes se posicionan ante la agenda, evitando así el debate 
parlamentario, pero exponiéndose a cuestiones comprometidas desde los medios. En este ámbito, la habilidad, carisma e imagen del compareciente son factores clave para el éxito de la acción del gobierno. Pero con la intervención de los medios en la configuración de la agenda, los gobiernos corren el riesgo de perder el control sobre ella en favor de aquéllos, por lo que la agenda quedará marcada por la competición entre los medios y el ámbito político.

Porras-Nadales pone de manifiesto que la progresiva "presidencialización" de las formas de gobierno encontraría acomodo en el contexto de la gobermedia. Indica que el sistema semipresidencial es el que más se adecúa a este modelo, ya que el presidente puede ejercer ese liderazgo personal propio de su cargo, mientras que el gobierno, liderado por su primer ministro, representaría la pura gestión gubernamental. En todo caso, puesto que los medios ponen su foco en unos asuntos, ocultando así otros, la trasparencia de este modelo no está ni mucho menos garantizada. Es indudable que este modelo presenta otro riesgo de "captura" entre el gobierno y las grandes corporaciones mediáticas. Según el autor, la intensa competitividad hace tambalear el ya precario consenso que existió en torno al Estado social.

Las diserciones finales del autor tratan la evolución dinámica de los modelos. Aunque la finalidad de la acción de gobierno apenas haya cambiado (conseguir el "bienestar sostenible"), sí lo han hecho los procedimientos. También se ha transformado la manera de determinar quién gobierna, y el Derecho como instrumento de la acción de gobierno, que se fue adaptando a los sucesivos modelos. Se abordan la retroalimentación y el control del sistema, como garantía para su reproducción, y como peligro por la "autorreproducción" indefinida de gobiernos que cuenten con el apoyo mediático además de sus propios recursos gubernamentales, en el contexto de la gobermedia, para lo que el politólogo ve necesaria la limitación de los mandatos. 